\title{
Swerving away from diabetic nephropathy by means of divine guidance
}

\author{
Friedrich C. Luft
}

Published online: 29 June 2013

(C) Springer-Verlag Berlin Heidelberg 2013

The netrins are a class of proteins involved in axon guidance. They are genetically conserved across nematodes, flies, amphibians, and mammals. Structurally, the netrins resemble the basement membrane protein, laminin. The netrins are chemotropic. Netrin attraction is mediated through "deleted in colorectal carcinoma" (cUNC-40/DCC) cell surface receptors, while repulsion requires UNC-5 receptors. Netrin-1 is found in many sites of the central nervous system as well as in the somatic mesoderm, pancreas, and cardiac muscle. More recent research has linked netrin to the development and formation of non-neural tissue, the detection of cancer, and other diseases. Even myocardial infarction, Alzheimer's disease, and kidney diseases have been found to involve netrins, particularly netrin-1 [1]. As a matter of fact, the kidney has one of the highest netrin-1 expressions. With ischemia/reperfusion, netrin1 protein expression in renal tubular cells increases further and urinary netrin-1 excretion increases accordingly [2]. Netrin-1's appearance in the urine suggested that netrin-1 might serve as an acute kidney injury "biomarker." A survey of 13 patients with acute kidney injury supported that point of view [3].

What is netrin-1 doing in the kidney? Further work by the same group initially exploring the role of netrin-1 in the kidney showed that netrin-1 increased renal tubular epithelial cell proliferation and migration through the UNC5B receptor. Moreover, the increase in cell proliferation, but not migration, was mediated via activation of Akt and ERK pathways [4]. How does netrin-1 work? To address that issue, Wang et al. studied a transgenic model overexpressing netrin-1 in the kidney [5]. Acute ischemia/reperfusion was the model they employed. Netrin-1 overexpression exerted a protective effect from acute kidney injury. Mechanisms included preservation of morphology, reduced cytokine expression, and reduced

F. C. Luft $(\bowtie)$

Experimental and Clinical Research Center, MDC/Charité,

Lindenbergerweg 80,13125 Berlin, Germany

e-mail: luft@charite.de oxidative stress in the kidney of transgenic mice, compared to wild-type mice. The authors concluded that netrin-1 protects renal tubular epithelial cells against ischemia reperfusioninduced injury by increasing proliferation of recovering cells and suppressing apoptosis in injured cells. However, mechanistically, these studies were insufficient.

Since the ischemic kidney is particularly prone to reperfusion-elicited inflammation, the group next sought to determine the function of netrin-1 and its receptor UNC5B in renal ischemia-reperfusion-induced inflammation [6]. They reasoned that ischemia/reperfusion injury rests on inflammatory processes. Renal ischemia-reperfusion caused a rapid decrease in serum netrin-1 levels. However, the administration of recombinant netrin-1, before or after renal ischemia-reperfusion, reduced kidney injury, resulted in apoptosis, and lowered monocyte and neutrophil infiltration. Furthermore, the cytokines IL- 6 , IL- $1 \beta$, and TNF- $\alpha$ and the chemokines MCP-1, macrophage-derived cytokine, monokine-induced IFN- $\gamma$, keratinocyte-derived chemokine, and chemokine with-6-cysteines production were reduced. The authors investigated different netrin-1 receptors on leukocytes. The cells showed very high expression of UNC5B but not UNC5C, UNC5D, neogenin, or the DCC receptor. The authors next blocked the UNC5B receptor. They found that neutralization of UNC5B receptor reduced netrin-1-mediated protection against renal ischemia-reperfusion injury. Blockade also increased monocyte and neutrophil infiltration, as well as serum and renal cytokine and chemokine production, with increased kidney injury and renal tubular cell apoptosis. These remarkable studies demonstrated that netrin-1, acting through UNC5B receptor, reduces renal ischemia-reperfusion injury and its associated renal inflammation.

The results caused the same group to inspect any role of netrin-1 in diabetic nephropathy. Mohamed et al. used tubule-specific transgenic mice overexpression nephrin [7]. They induced type 1 diabetes with streptozotocin. Diabetes was associated with increased infiltration of neutrophils and 
macrophages, chemokine expression, and tubular epithelial cell apoptosis in kidney. In contrast, the changes were minimal in the kidney of netrin-1 transgenic mice. Diabetes also induced a large increase in the excretion of prostaglandin E2 (PGE2) in urine, which was suppressed in netrin-1 transgenic mice. Netrin-1-induced suppression of PGE2 production was mediated through suppression of NF- $\mathrm{kB}-$ mediated cyclooxygenase-2 in renal tubular epithelial cells. Furthermore, netrin-1 also increased albumin uptake by proximal tubular epithelial cells through the PI3K and ERK pathways without increasing glucose uptake. The authors' findings suggest that netrin- 1 is a major regulator of inflammation and apoptosis in diabetic nephropathy and may be a useful therapeutic molecule for treating chronic kidney diseases such as diabetic nephropathy.
Thus far, the netrin-1-kidney story has been dominated by one laboratory [2-7]. Confirmatory information from an independent source is helpful, particularly if the model is a different one and the findings are not quite the same. Tak et al. [8] investigated a protective role for netrin-1 during diabetic nephropathy. They studied the role of netrin-1 during diabetic nephropathy, by inducing diabetes in mice with streptozotocin treatment. Initially, they observed that wildtype mice demonstrated robust increases in renal, urinary, and plasma netrin-1 protein levels during diabetic nephropathy. They then investigated mice with partial netrin-1 deficiency (Ntrn1+/- mice); Ntrn1-/- mice die in utero. Ntrn1+ I- mice developed more severe diabetic nephropathy, including more severe loss of kidney function. Tak et al. [8] subsequently performed pharmacologic studies with

\section{STRING Network connecting NTN1 to ADORA2B}

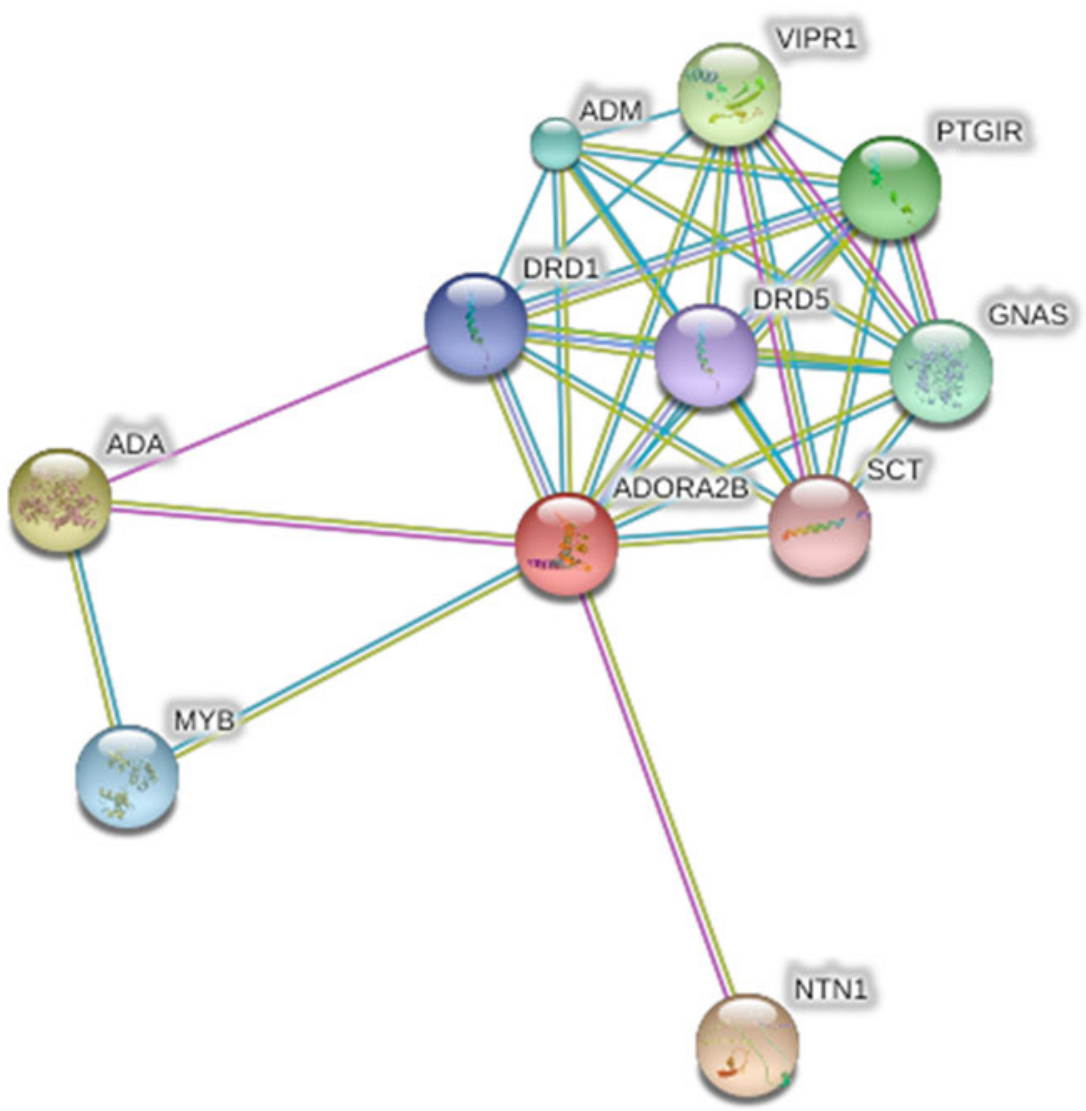

Fig. 1 The adenosine $\mathrm{A} 2 \mathrm{~b}$ receptor, receptor for adenosine, and $A D O R A 2 B$ signals by $\mathrm{G}$ proteins, which activate adenylyl cyclase. The process may be in part regulated by netrin-1. NTN1 encodes netrin-1. The MYB gene encodes the Myb proto-oncogene protein, a transcriptional activator. The $A D A$ gene encodes adenosine deaminase. Present in virtually all mammalian cells, ADA's primary function in humans is the development and maintenance of the immune system. $S C T$ encodes secretin, which belongs to the glucagon protein family. DRD5 encodes the $\mathrm{D}(1 \mathrm{~B})$ dopamine receptor. DRD1 encodes the dopamine receptor D1. GNAS encodes a stimulatory G protein alpha subunit (Gs- $\alpha$ ), a key component of many signal transduction pathways. Prostacyclin receptor (or prostaglandin I2 receptor, IP1) is a receptor for prostacyclin. The receptor is encoded by the human gene PTGIR. The VIPIR gene encodes vasoactive intestinal polypeptide receptor 1 also known as VPAC1. Adrenomedullin is a peptide associated with pheochromocytoma - a tumor arising from adrenal medulla, encoded by the ADM gene. Network was obtained from STRING 9.0. (http://string90.embl.de/newstring_cgi/show_network_section.pl? identifier=9606.ENSP00000173229\&all_channels_on=1 $\&$ network flavor=evidence\&targetmode= proteins) 
recombinant netrin-1 treatment given continuously via osmotic pump. They found that netrin-1 treatment was associated with attenuated albuminuria and improved histologic scores for diabetic nephropathy, compared to controls. They next considered earlier studies implicating purinergic signaling in netrin-1-elicited tissue protection. They observed that mice deficient in the adenosine $\mathrm{A}_{2 \mathrm{~B}}$ receptor (Adora2b) were not protected when exposed to netrin-1 treatment. ADORA2B is a $\mathrm{G}$ protein-coupled adenosine receptor. ADORA2B is an integral membrane protein that stimulates adenylate cyclase activity in the presence of adenosine. The protein also interacts with netrin-1 and its involvement in axon elongation. The results suggest a functional role for endogenous netrin-1 in attenuating diabetic renal disease. The studies of Mohamed et al. [7] and Tak and colleagues [8] seem quite complimentary. "One swallow does not make a summer," but two swallows probably fly in the right direction. Tak et al. add the implication that ADORA2b is involved in signaling the effects of netrin-1. A network connecting netrin-1 to ADORA2b is shown in Fig. 1. This research direction represents a beginning, not an end. Hopefully, this interesting body of work will spur mechanistic studies on netrin-1 and renal disease further. Clearly, such a state-of-affairs would be better than allowing netrin-1 to languish solely as a biomarker [9].

Respectfully, Friedrich C. Luft

\section{References}

1. Rajasekharan S, Kennedy TE (2009) The netrin protein family. Genome Biol 10:239

2. Wang W, Reeves WB, Ramesh G (2008) Netrin-1 and kidney injury. I. Netrin-1 protects against ischemia-reperfusion injury of the kidney. Am J Physiol Ren Physiol 294:F739-F747

3. Reeves WB, Kwon O, Ramesh G (2008) Netrin-1 and kidney injury, II. Netrin-1 is an early biomarker of acute kidney injury. Am J Physiol Ren Physiol 294:F731-F738

4. Wang W, Reeves WB, Ramesh G (2009) Netrin-1 increases proliferation and migration of renal proximal tubular epithelial cells via the UNC5B receptor. Am J Physiol Ren Physiol 296:F723-F729

5. Wang W, Reeves WB, Pays L, Mehlen P, Ramesh G (2009) Netrin-1 overexpression protects kidney from ischemia reperfusion injury by suppressing apoptosis. Am J Pathol 175:1010-1018

6. Tadagavadi RK, Wang W, Ramesh G (2010) Netrin-1 regulates Th1/ Th2/Th17 cytokine production and inflammation through UNC5B receptor and protects kidney against ischemia-reperfusion injury. $\mathrm{J}$ Immunol 185:3750-3758

7. Mohamed R, Jayakumar C, Ranganathan PV, Ganapathy V, Ramesh G (2012) Kidney proximal tubular epithelial-specific overexpression of netrin-1 suppresses inflammation and albuminuria through suppression of COX-2-mediated PGE2 production in streptozotocininduced diabetic mice. Am J Pathol 181:1991-2002

8. Tak E, Ridyard D, Badulak A, Giebler A, Shabeka U, Werner T, Clambey E, Moldovan R, Zimmerman MA, Elzschig HK et al (2013) Protective role for netrin-1 during diabetic nephropathy. J Mol Med. doi:10.1007/s00109-013-1041-1

9. Mussap M, Noto A, Fravega M, Fanos V (2011) Urine neutrophil gelatinase-associated lipocalin (UNGAL) and netrin-1: are they effectively improving the clinical management of sepsisinduced acute kidney injury (AKI)? J Matern Fetal Neonatal 24(Suppl 2):15-17 